\title{
Computer-Assisted Smoking Cessation Intervention
}

National Cancer Institute

\section{Source}

National Cancer Institute. Computer-Assisted Smoking Cessation Intervention. NCI

Thesaurus. Code C116524.

The use of a computer for the creation and execution of a plan to help an individual stop smoking. 